\title{
A NOTE ON DERIVATIVES AND NEIGHBORLY FUNCTIONS
}

\author{
ABRAM V. MARTIN
}

1. Introduction. Let $S$ and $T$ be metric spaces and let $d$ and $d^{\prime}$ denote the distance functions in $S$ and $T$, respectively. Let $f$ be a function on $S$ to $T$. In [1], Bledsoe defined $f$ to be neighborly at the point $x$ of $S$ if and only if for every $\epsilon>0$ there exists a nonempty open sphere $U$ of $S$ such that, for every $y$ in $U, d(x, y)+d^{\prime}(f(x), f(y))<\epsilon$. If $f$ is neighborly at every point of $S$, then we say that $f$ is neighborly over $S$, or simply that $f$ is neighborly. The concept of a neighborly function is a generalization of the concept of a continuous function, since the open sphere $U$ is not required to contain the point $x$.

Bledsoe proved [1] that if $g$ is a function which is the limit of a sequence of neighborly functions, then the set of points of discontinuity of $g$ is of the first category. Obviously, then, this conclusion holds if $g$ itself is neighborly. This result suggests that there might be a close relationship between neighborly functions and derivatives, and that the concept of a neighborly function might be helpful in connection with the unsolved problem of finding an intrinsic characterization of those functions which are derivatives. The purpose of this note is to prove the theorem below, which offers some prospect of being useful in characterizing derivatives. ${ }^{1}$

Although the theorem was suggested by Bledsoe's results, it is not necessary to be familiar with his paper in order to understand this note.

2. Whenever we speak of a function of a real variable we shall mean a function whose domain of definition is the set of all real numbers. We shall need the following lemma.

LEммA. If $f$ is a Baire class I real-valued function of a real variable, $s<t$, and $P$ is any nonempty closed subset of the reals, then there is an open interval $J$ such that $J P$ is nonempty and either $J P \subset \mathrm{Ex}(f(x)>s)$ or $J P \subset \operatorname{Ex}(f(x)<t)$.

Proof. By a theorem due to Lebesgue [5, p. 128], the proof of

Presented to the Society, November 17, 1956; received by the editors June 15, 1956 and, in revised form, August 30, 1956.

1 The author is indebted both to the referee and to D. W. Dubois for pointing out simplifications in the proof of the theorem. He is also indebted to D. W. Dubois for calling his attention to the example from Hobson which is cited in the Remarks. 
which is straightforward, each of the sets $A_{1}=P \cap \operatorname{Ex}(f(x)>s)$ and $A_{2}=P \cap \operatorname{Ex}(f(x)<t)$ is a countable union of closed sets. Clearly $P=A_{1} \cup A_{2}$. Since $P$ is a complete metric space, it follows by Baire's Density Theorem that either $A_{1}$ or $A_{2}$ contains a nonempty open subset of $P$. Hence the lemma follows.

THEOREM. If $F$ is any real-valued function of a real variable whose derivative $F^{\prime}$ is defined everywhere and is Riemann integrable over every finite interval, then $F^{\prime}$ is neighborly.

Proof. Let $F^{\prime}(x)=f(x)$. The theorem is proved by assuming that $f$ is not neighborly at $x_{1}$ and obtaining a contradiction. There is clearly no loss of generality in assuming $f\left(x_{1}\right)=0$. From the definition of a neighborly function and the assumption that $f$ is not neighborly at $x_{1}$, it follows that there is a closed interval $I_{1}$ which contains $x_{1}$ in its interior, and a positive number $\epsilon$, such that at every point $x$ of continuity of $f$ lying in $I_{1},|f(x)| \geqq \epsilon$. We assume, without loss of generality, that $|f(x)| \geqq 3$ for every point of continuity in $I_{1}$, since this can be achieved by multiplying by the constant $3 / \epsilon$.

Since $f$ is a derivative it is of Baire class $I$, and hence the set $B$ of points of continuity of $f$ is dense. Hence we can choose a closed interval $I$, contained in $I_{1}$ and containing $x_{1}$ in its interior, such that the end points of $I$ are points of $B$. Let $M$ be the set of those real numbers $x$ for which $|f(x)| \geqq 2$. Let $D$ be the interior of $M$ and let $P$ $=I-D$. If $x$ is in $B I$, then $|f(x)| \geqq 3$ and hence $x$ is in an open interval at every point $y$ of which, $|f(y)| \geqq 2$. Hence $B I \subset D$. Clearly $P$ is a closed subset of the interior of $I$. Also $P$ is nonempty since $x_{1}$ in is $P$.

Let $K=\mathrm{E} x(f(x)>-1)$ and $Q=\mathrm{E} x(f(x)<1)$. By our lemma there is an open subinterval $J$ of $I$ such that $J P$ is nonempty and either $J P \subset K$ or $J P \subset Q$. By changing the sign of $f$, if necessary, we see that there is no loss of generality in assuming $J P \subset K$.

Since $f$ is a derivative it satisfies the Darboux condition: given a number $u$ such that $f(b)<u<f(c)$, there exists a number $d$ between $b$ and $c$ such that $f(d)=u[2$, p. 117].

From the fact that $J=J P \cup J D$ we have for each $x$ in $J$ either $f(x)>-1$ or $|f(x)| \geqq 2$. Hence there is no $x$ in $J$ for which $f(x)=-1$, and therefore by the Darboux principle there is no $x$ in $J$ for which $f(x) \leqq-2$. Consequently for every $x$ in $J D, f(x) \geqq 2$. Since $B I \subset D$ and $J \subset I$ it follows that $B J \subset J D$. Since $f$ is Riemann integrable, the set $B J$, and hence its superset $J D$, have relative measure 1 in $J$. Thus $f(x) \geqq 2$ over a set of relative measure 1 in $J$. Since $J P$ is nonempty, there is a point $p$ in $J$ for which $f(p)<2$. Furthermore if $x$ is in $J$ and $x>p$ then 


$$
F(x)-F(p)=\int_{p}^{x} f(t) d t \geqq 2(x-p)
$$

[4, Theorem 11.83]. Hence we have

$$
f(p)=F^{\prime}(p)=\lim _{x \rightarrow p+} \frac{F(x)-F(p)}{x-p} \geqq 2 .
$$

This contradicts the assertion that $f(p)<2$, and completes the proof of the theorem.

3. Remarks. The condition in the theorem that $F^{\prime}$ be Riemann integrable can not be dropped. Hobson [3, pp. 412-421] describes a function $F$ which has a derivative $F^{\prime}$ which is finite everywhere, but which has the following properties: both the set of points of discontinuity of $F^{\prime}$, and also the set of points of continuity, are everywhere dense, and at every point $x$ of continuity of $F^{\prime}, F^{\prime}(x)=0$. In view of these properties it is easy to show that $F^{\prime}$ is not neighborly.

The converse of the theorem is not true. If $f(x)=0$ for $x \leqq 0$ and $f(x)=1$ for $x>0$, then $f$ is Riemann integrable and neighborly but is not a derivative.

\section{REFERENCES}

1. W. W. Bledsoe, Neighborly functions, Proc. Amer. Math. Soc. vol. 3 (1952) pp. 114-115.

2. P. Franklin, A treatise on advanced calculus, New York, 1940.

3. E. W. Hobson, The theory of functions of a real variable and the theory of Fourier's series, vol. 2, Washington, 1950.

4. E. C. Titchmarsh, The theory of functions, Oxford, 1932.

5. C. de La Vallée Poussin, Intégrales de Lebesgue, fonctions d'ensemble, classes de Baire, Paris, 1950.

University of New MeXico 